$4-2009$

\title{
A Real-Time, 3-D Musculoskeletal Model for Dynamic Simulation of Arm Movements
}

\author{
Edward K. Chadwick \\ Case Western Reserve University \\ Dimitra Blana \\ Case Western Reserve University
}

Antonie J. van den Bogert

Cleveland State University, a.vandenbogert@csuohio.edu

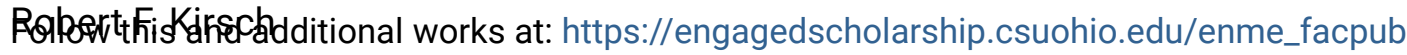
Sose Western Reserve University

Part of the Biomechanical Engineering Commons, and the Biomedical Engineering and Bioengineering

\section{Commons}

How does access to this work benefit you? Let us know!

\section{Publisher's Statement}

(C) 2009 IEEE. Personal use of this material is permitted. Permission from IEEE must be obtained for all other uses, in any current or future media, including reprinting/republishing this material for advertising or promotional purposes, creating new collective works, for resale or redistribution to servers or lists, or reuse of any copyrighted component of this work in other works.

\section{Original Citation}

Chadwick, E. K., Blana, D., van den Bogert, A. J., and Kirsch, R. F., 2009, "A Real-Time, 3-D Musculoskeletal Model for Dynamic Simulation of Arm Movements," Biomedical Engineering, IEEE Transactions on, 56(4) pp. 941-948.

This Article is brought to you for free and open access by the Mechanical Engineering Department at EngagedScholarship@CSU. It has been accepted for inclusion in Mechanical Engineering Faculty Publications by an authorized administrator of EngagedScholarship@CSU. For more information, please contact library.es@csuohio.edu. 


\title{
A Real-Time, 3-D Musculoskeletal Model for Dynamic Simulation of Arm Movements
}

\author{
Edward K. Chadwick*, Dimitra Blana, Antonie J. (Ton) van den Bogert, and Robert F. Kirsch, Member, IEEE
}

\begin{abstract}
Neuroprostheses can be used to restore movement of the upper limb in individuals with high-level spinal cord injury. Development and evaluation of command and control schemes for such devices typically require real-time, "patient-in-the-loop" experimentation. A real-time, 3-D, musculoskeletal model of the upper limb has been developed for use in a simulation environment to allow such testing to be carried out noninvasively. The model provides real-time feedback of human arm dynamics that can be displayed to the user in a virtual reality environment. The model has a 3-DOF glenohumeral joint as well as elbow flexion/extension and pronation/supination and contains 22 muscles of the shoulder and elbow divided into multiple elements. The model is able to run in real time on modest desktop hardware and demonstrates that a large-scale, 3-D model can be made to run in real time. This is a prerequisite for a real-time, whole-arm model that will form part of a dynamic arm simulator for use in the development, testing, and user training of neural prosthesis systems.
\end{abstract}

Index Terms-Biomechanics, functional electrical stimulation (FES), musculoskeletal modeling, shoulder, simulation, upper limb.

\section{INTRODUCTION}

$\mathbf{F}$ UNCTIONAL electrical stimulation (FES) can be used to restore upper limb function in people with high-level spinal cord injury (SCI) through the electrical stimulation of nerves and muscles to generate movement in the paralyzed limb. Upper limb FES systems have been described by numerous groups [1]-[5], but have more commonly been applied to people with midcervical level (C5-C6 SCI) or thoracic-level injuries [6]. People with high-level (C1-C4 SCI) injuries have fewer options for command sources for FES than those with lower level injuries, who can often make use of retained voluntary function to control an FES-based neuroprosthesis.
Operating an FES system requires sophisticated control of a multiple-DOF, kinematically coupled system of joints using redundant, nonlinear actuators from a limited, artificial command source. For goal-directed movements in the upper extremity, feedback control involving user input is essential, and the command interface for the user becomes an integral part of the control system. Musculoskeletal models have been used successfully to help design control systems for FES by a number of authors [7]-[10]. These modeling studies have consisted of offline analyses of model and controller behavior, not realtime simulations. They did not generate real-time feedback for the user and did not allow the adaption of control signals in real time. The execution of "patient-in-the-loop" experiments in which it is possible to give the subject real-time feedback of simulated arm movement requires a virtual reality environment with real-time simulation of arm dynamics.

A brain-machine interface (BMI) is an example of such a command interface and offers a promising source of commands for neuroprosthetic control, but to date, BMI research has tended to focus on the control of nondynamic tasks such as 2-D computer mouse movement [11], or in some cases simulated 3-D target reaching tasks [12]. For the use of brain signals for real arm control (i.e., with a neuroprosthesis), it may be essential to consider the effect of arm and muscle dynamics on the nature of the extracted signal. Carmena et al. [13], for example, showed movement control with a BMI by decoding multiple movement parameters including hand force and muscle activations, not just movement trajectories.

In order to help answer the question of whether arm dynamics affect the nature of the required movement command and facilitate FES controller development, a tool for the real-time, dynamic simulation of arm movement would be very useful. A number of large-scale models of the upper limb have been described in the literature [14]-[17], but the focus of these models has always been anatomical fidelity, and not simulation speed. Real-time simulation was not a goal of these studies, and no data are given on simulation speeds. Other groups have described very promising simulation environments that do feature real-time simulation, incorporating musculoskeletal modeling and visualization [18], but to date, details on the complexity of models that can be simulated in real time are not available. Davoodi et al. [19] described the virtual reality aspects of the simulation environment in some detail, but did not provide many details on the biomechanical modeling aspects.

The long-term goal of this paper, therefore, was to develop a complex, 3-D biomechanical model of the upper limb that runs in real time. The model described simulates realistic arm dynamics (inertia, kinematic coupling, muscle dynamics) for use in the 
TABLE I

ANGULAR LIMITS FOR EACH DEGREE OF FREEDOM (IN DEGREES)

\begin{tabular}{|c||c||c|}
\hline Degree of freedom & Min angle & Max angle \\
\hline Plane of elevation & -90 & 90 \\
Angle of elevation & 5 & 90 \\
Internal rotation & -55 & 70 \\
Elbow flexion & 5 & 140 \\
Forearm pronation & 5 & 160 \\
\hline
\end{tabular}

so-called dynamic arm simulator (DAS). The real-time dynamics model interfaces with a visualization environment developed using Game Studio (Conitec Data Systems, Inc.). This gives the user of the simulator direct visual feedback regarding the movement of their virtual arm and allows the development of neural prosthesis controllers and BMIs, as well as providing a training environment for potential users of such systems. The model is fully customizable, e.g., allowing compromises in muscle strength associated with SCI to be accurately modeled. The clinical relevance of this paper is that it allows rapid development of rehabilitation technologies for people with SCI or other neurological impairment.

The aim of this current study was to develop a dynamic model of the human upper limb that would run in real time and be of sufficient complexity to allow realistic simulations of arm movement. This real-time model is a prerequisite for the DAS that could be used in the development, testing, and user training of neural prosthesis systems.

\section{METHODS}

\section{A. Musculoskeletal Model in SIMM}

The structure of the model was initially built using software for interactive musculoskeletal modeling (SIMM) (MusculoGraphics, Inc.), a graphical musculoskeletal modeling package. The model has 5-DOF: 3 at the glenohumeral joint, 1 for elbow flexion/extension, and 1 for pronation/supination. The scapula was considered to be the base of this model and was fixed. The glenohumeral joint was modeled as three orthogonal hinges or pin joints as they are known in SIMM. The elbow flexion axis and the forearm pronation axis were also modeled as pin joints, with direction vectors determined by cadaver kinematic measurement [20]. Limits on the range of motion of the joints were imposed based on data from Gûnal et al. [21], who measured the range of motion of the upper limb in a large number of male subjects. These angles were restricted slightly where necessary in order to ensure the correct wrapping of muscles in the SIMM model, and the final values are shown in Table I. The range of motion of the humerus with respect to the thorax is, of course, reduced compared to a full upper limb model due to the fixed scapula.

Muscles crossing joints proximal to the glenohumeral joint have the correct line of action and wrapping, but are considered to originate from the scapular rigid body. A total of 22 muscles and muscle parts are included in the real-time model, divided into 102 muscle elements that are modeled independently. Muscles are modeled using the minimum number of elements needed to accurately model the mechanical line of action of each part.
TABLE II

Muscles INCLuded in ReAL-Time Model, SHOWIng JoInts CROSSED BY EACH MUSCLE (GLENO-HUMERAL: GH, HUMERO-ULNAR: HU, OR RADIO-ULNAR: RU) AND NUMBER OF ELEMENTS USED TO MODEL MUSCLE

\begin{tabular}{|l||c||c|}
\hline Muscle & Joints & No. of elements \\
\hline Deltoid, scapular part & GH & 11 \\
Deltoid, clavicular part & GH & 4 \\
Coracobrachialis & GH & 3 \\
Infraspinatus & GH & 6 \\
Teres minor & GH & 3 \\
Teres major & GH & 4 \\
Supraspinatus & GH & 4 \\
Subscapularis & GH & 11 \\
Biceps, long head & GH, HU and RU & 1 \\
Biceps, short head & GH, HU and RU & 2 \\
Triceps, long head & GH and HU & 4 \\
Latissimus dorsi & GH & 6 \\
Pectoralis major, thoracic part & GH & 6 \\
Pectoralis major, clavicular part & GH & 2 \\
Triceps, medial head & HU & 5 \\
Brachialis & HU & 7 \\
Brachioradialis & HU and RU & 3 \\
Pronator teres & HU and RU & 2 \\
Supinator & HU and RU & 5 \\
Pronator quadratus & HU & 3 \\
Triceps, lateral head & & 5 \\
Anconeus & HU & 5 \\
\hline
\end{tabular}

In the case of widely diverging muscles such as the deltoids, this was as many as 11 elements. The number of elements used for each muscle and the degrees of freedom crossed by the muscles are shown in Table II.

Geometrical data for the real-time model were taken from the cadaver studies of Klein-Breteler et al. [20]. In these studies, the muscles and muscle parts controlling the movement of the shoulder and elbow were divided into multiple elements and their origins and insertions measured. Joint surfaces and other bony contours were digitized for modeling using geometrical forms, and an extensive set of muscle architecture parameters was measured. This included tendon length, physiological crosssectional area (PCSA), pennation angle, sarcomere length, and fiber length. All these data are available via the International Shoulder Group Web site. ${ }^{1}$

\section{B. Equations of Motion}

The equations of motion were derived using SDFAST (Symbolic Dynamics and Parametric Technology Corporation, Needham, MA). The dynamics export feature of SIMM was used to form the model description file for SDFAST, model.sd. This file was processed by SDFAST to generate the equations of motion as $\mathrm{C}$ code for use in the simulation. The equations of motion are expressed as second-order differential equations of the following form:

$$
M(q) \ddot{q}=Q_{M}(q, \dot{q}, t)+Q_{E}(q, \dot{q})+Q_{C}(q, \dot{q})
$$

where $M$ is the mass matrix, $q$ 's are the generalized coordinates, and $Q_{M}, Q_{E}$, and $Q_{C}$ are the generalized force terms due to muscles, external forces, and coriolis and centrifugal forces.

\footnotetext{
${ }^{1}$ http://internationalshouldergroup.org.
} 


\section{Muscle Wrapping and Lines of Action}

Muscle wrapping objects were defined in the SIMM file to allow the calculation of the lines of action and the moment arms of the muscles in all positions of the model. These were based on the geometrical structures measured in the cadaver studies described before [20] and used in [22], and included spheres for the humeral head, an ellipsoid for the thorax, and cylinders for the humerus, ulna, and radius. The run-time calculation of muscle wrapping, however, is too time-consuming for a real-time application. In this study, preprocessing of the muscle moment arms and lines of action was carried out to allow faster operation at run time.

Muscle moment arms were exported from SIMM for each muscle element crossing each degree of freedom over the whole range of model motions. In the real-time model, muscleskeleton coupling was represented by a polynomial model for muscle length as a function of kinematic degrees of freedom. This was done in preference to fitting the moment arms directly as it reduces the number of model coefficients needed, thereby decreasing simulation time. This also avoids overfitting the data, which can happen when fitting the moment arms directly, resulting in the model not being mechanically consistent. The moment arm data from the SIMM model were then used to assess the fitting accuracy. Polynomial terms were added to the model until the moment arm error for each element was less than $10 \%$ of the maximum moment arm value or $2 \mathrm{~mm}$, whichever was the greater. This gave a good compromise between accurately describing the moment arms and limiting the number of polynomial terms needed for that description. A similar procedure was followed to approximate the muscle lines of action. In the case of the lines of action, one polynomial was required for each orthogonal component of the path. The lines of action of the muscles were needed for the calculation of the joint reaction force and subsequently the assessment of glenohumeral stability described in Section II-E.

\section{Muscle Model}

A three-component, Hill-type muscle model was used to model muscle force generation. The model consists of a contractile element for active force generation, a series elastic element (SEE) representing tendon and other series stiffnesses, and a parallel elastic element (PEE) representing the passive stiffness of the muscle belly. Activation dynamics were governed by the first-order differential equation

$$
\dot{a}(t)=\left(c_{1} u(t)+c_{2}\right)(u(t)-a(t))
$$

where $a$ is the active state, $u$ is the neural command, and the terms $c_{1}$ and $c_{2}$ are chosen to give activation and deactivation time constants derived from the proportions of fast and slow twitch fibers in the muscle (derived from [23]).

The contractile element produces a force $F_{\text {ce }}$, which is dependent on the shortening velocity $V_{\text {ce }}$, fiber length $L_{\text {ce }}$, and active state $a$

$$
F_{\text {ce }}=f\left(L_{\text {ce }}\right) g\left(V_{\text {ce }}, a\right) a
$$

where $f$ describes the force-length relationship and $g$ describes the Hill shortening equation (for details see McLean et al. [24]). In order to prevent infinite muscle velocities at zero activation, a passive damper of $10 \mathrm{~N} / \mathrm{m} \cdot \mathrm{s}^{-1}$ was modeled in parallel with the contractile element for low activations (less than 0.02). This value was within the range found by Kirsch et al. [25] and allowed the arm to fall at a realistic rate.

Stiffness properties for the series and parallel elastic components were given by quadratic relationships between force and elongation (where $L_{\text {slack }}$ is the element slack length normalized to the optimal fiber length)

$$
F=k\left(L-L_{\text {slack }}\right)^{2} .
$$

The stiffness parameter $k$ for the SEE was set such that $4 \%$ elongation was achieved when the maximal isometric force was applied (from [24]). $L_{\text {slack }}$ for the series elastic element was approximated by the tendon slack length measured in the cadaver studies. $L_{\text {slack }}$ for the PEE is a harder parameter to estimate, as data for doing this are not available. Following the example of McLean et al. [24], a default value of 1.0 was used (normalized to optimum fiber length), except for a small number of muscle elements for which this led to prohibitively high passive forces. For these elements, the value of $L_{\text {slack }}$ was estimated based on the desired range of motion of the joint that the muscle crossed. There are insufficient data in the literature to obtain this value in any other way. These values are shown in Table $\mathrm{V}$ in the Appendix.

\section{E. Computation of GH Stability}

The glenohumeral joint is minimally stabilized by passive structures around the joint and requires active stabilization from the rotator cuff muscles during movement. The ability to monitor the stability of that joint is essential in the design of controllers for FES systems, a major application of this model.

At each major time step of the simulation, the resultant force vector between the humeral head and the glenoid was calculated. This was the vector sum of all contributions to the joint reaction force: external forces on the limb as well as the sum of all muscle forces for muscles crossing the glenohumeral joint. The stability of the glenohumeral joint is defined as a function of the angle of the resultant force in the glenoid relative to the maximum angle that can be reached before dislocation of the joint. Specifically, the stability value is defined as

$$
\mathrm{GH}_{\text {stab }}=\left(\frac{\theta}{\theta_{a}}\right)^{2}+\left(\frac{\phi}{\phi_{a}}\right)^{2}
$$

where $\theta$ and $\phi$ are the angles of the vector away from the normal to the glenoid along the major and minor axes of the ellipse, and $\theta_{a}$ and $\phi_{a}$ are the angles of that vector as it reaches the rim of the glenoid. The value of $\mathrm{GH}_{\mathrm{stab}}$ thus reaches 1 as the reaction force vector reaches the edge of the ellipsoid defining the glenoid fossa and has a value of zero when the vector is exactly in the center of the fossa. Values of less than 1 indicate that the force is inside the rim of the fossa and the joint is stable, 
TABLE III

Minimum ACTIVATIONS NECESSARY IN RotATOR CUFF MUSClES TO ENSURE STABILITY OF GLENO-HUMERAL JOINT WITH A FACTOR OF SAFETy OF 2

$$
\left(\mathrm{GH}_{\mathrm{stab}}<0.5\right)
$$

\begin{tabular}{|c||c||c||c|}
\hline Infraspinatus & Teres minor & Supraspinatus & Subscapularis \\
\hline 0.04 & 0.02 & 0.02 & 0.07 \\
\hline
\end{tabular}

and values greater than 1 indicate that the reaction force vector would point outside the fossa, tending to cause dislocation of the joint.

\section{F. Simulink and $x P C$ Target}

In order to guarantee real-time operation of the model, Matlab's xPC Target operating system was used. This is a specialized application of the Real-Time Workshop allowing fast development of real-time applications on x86 hardware. The model is developed in Simulink in simulation mode before being compiled as a real-time executable for execution on the real-time operating system (RTOS). This approach allows rapid development of code, maximizing model performance on given hardware as well as guaranteeing real-time operation.

When running a model on the real-time system xPC Target, it is required to use a fixed-step solver to guarantee real-time operation. The time taken for one complete integration of the system is reported as the task execution time (TET) by xPC Target. The minimum step size that can be used with a given model is this TET (plus a small amount to allow for fluctuations in the execution time). If the simulation is stable with that step size for a given integrator, or solver, then real-time operation is possible.

In this study, two solvers were compared for their performance and stability: the first-order Euler solver and the fourthorder Runge-Kutte solver.

\section{G. Muscle Stimulation Experiments}

Simulations of simple muscle stimulation experiments were carried out to test the model response to activation of the various muscle groups. Groups of muscles were activated sequentially in the model, and the resulting motions were observed. During these simulations, the TET for the model was recorded in order to assess the real-time capability of the model during simulated motions.

Table III shows the baseline activations used during the simulations. These are the minimum activations necessary to ensure stability of the glenohumeral joint in the resting position. Only the rotator cuff muscles (infraspinatus, teres minor, supraspinatus, and subscapularis) are active in this state, and the maximum of these activations is 0.07 . These values were found by iteratively increasing the values of the activations until a combination was found that reduced the value of the glenohumeral stability constraint $\left(\mathrm{GH}_{\mathrm{stab}}\right)$ to less than 0.5 , giving a factor of safety of 2 , and allowed the arm to hang in a neutral position (i.e., without excessive internal or external rotation). As defined previously, $\mathrm{GH}_{\text {stab }}$ must be less than 1 to ensure stability of the joint. (a)

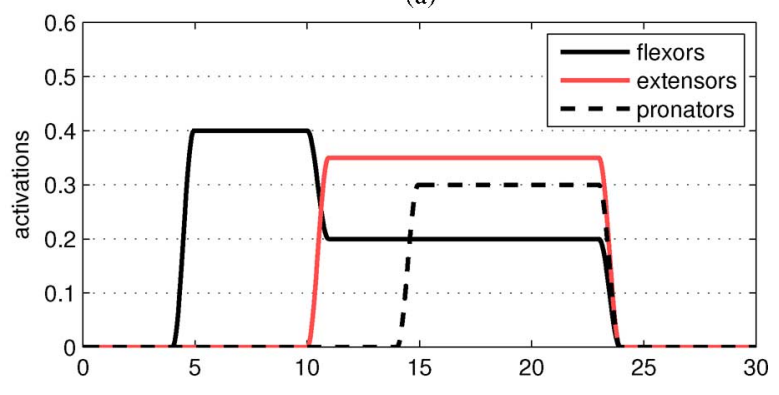

(b)

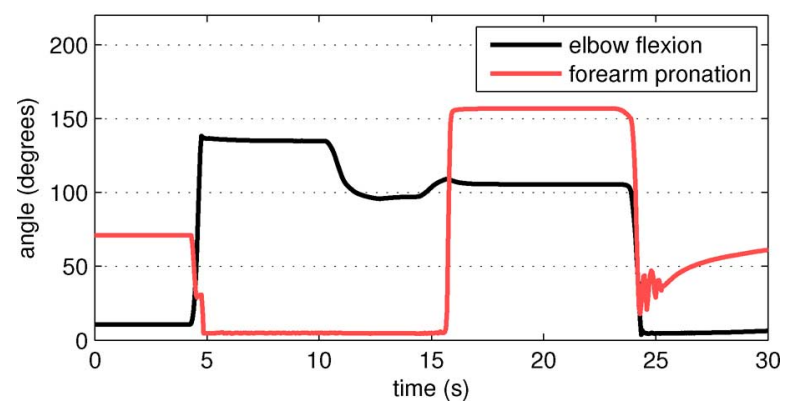

Fig. 1. (a) Activation of the elbow flexor muscles (at $4 \mathrm{~s}$ ), cocontraction of the flexors and extensors (at $10 \mathrm{~s}$ ), and activation of the flexors, extensors, and pronators (at $14 \mathrm{~s}$ ). (b) Flexion of the elbow to $140^{\circ}$ combined with full supination, extension back to $100^{\circ}$ followed by pronation to $160^{\circ}$. Finally, deactivation of all muscles allows the model to return to its initial position of $5^{\circ}$ flexion and $70^{\circ}$ pronation.

Activation of the various muscle groups to produce movement was then applied on top of these baseline activations. Three sets of activations were defined. The first tested the elbow flexion/extension and forearm pro/supination response of the model. The second tested the humeral abduction response by elevating the arm in the scapular plane, and the third repeated this motion, but increased the rotator cuff activity to stabilize the glenohumeral joint.

\section{RESULTS}

Fig. 1 shows the effect on the model of activation of combinations of elbow flexors (biceps brachii, brachialis, brachioradialis), extensors (triceps), and pronators (pronator teres and pronator quadratus). Part (a) shows activation of the elbow flexor muscles (at $4 \mathrm{~s}$ ), cocontraction of the flexors and extensors (at $10 \mathrm{~s}$ ), and activation of the flexors, extensors, and pronators (at $14 \mathrm{~s}$ ). Part (b) shows the movements produced by the activations applied in part a: flexion of the elbow to $140^{\circ}$ combined with full supination, then extension back to $100^{\circ}$, and then pronation of the forearm to $160^{\circ}$. Finally, deactivation of all muscles allows the model to return to its initial position of $5^{\circ}$ flexion and $70^{\circ}$ pronation.

Fig. 2 shows the effect of activating humeral abductors (the middle and anterior parts of the deltoids) (part a) in addition to the constant rotator cuff muscle baseline activations. The resulting motion of the model (part b) shows abduction of the humerus in approximately the scapular plane (part way between 
(a)

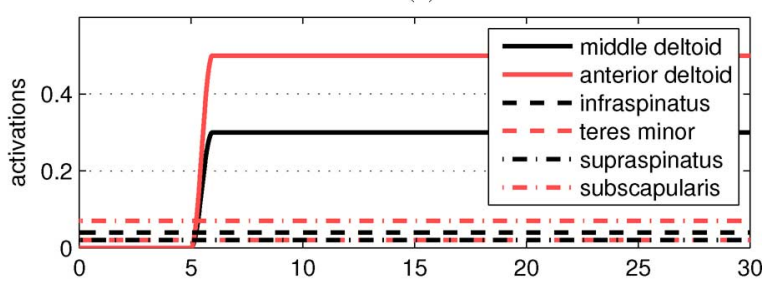

(b)

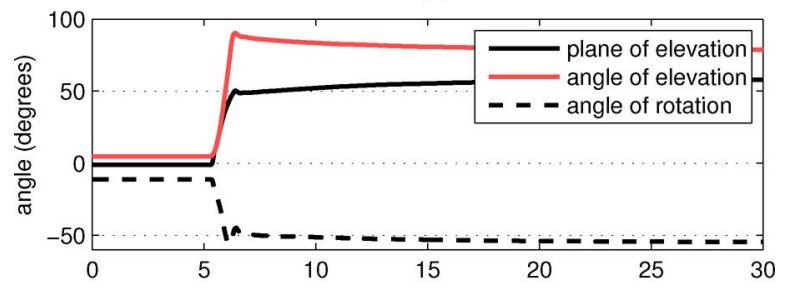

(c)

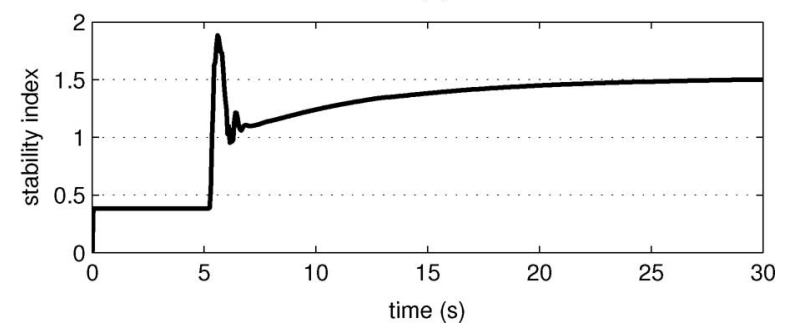

Fig. 2. (a) Activation of the middle and anterior parts of the deltoid muscles, and part (b) the model motions in response to these activations Rotator cuff activity was held at the baseline level throughout the movement, and part (c) shows the increase in the value of $\mathrm{GF}_{\mathrm{stab}}$ to greater than 1, indicating potential dislocation of the joint.

lateral abduction and forward flexion). The activity of the rotator cuff muscles was not adjusted during these motions, and part (c) of the figure shows the increase in the value of $\mathrm{GH}_{\text {stab }}$ to greater than 1 , indicating instability of the glenohumeral joint.

Fig. 3 shows the same deltoid activity as the previous figure, but this time with a concurrent increase in the rotator cuff activity. This level of activation was chosen merely to be sufficient to maintain glenohumeral stability. The resulting motion is quite similar with slightly increased humeral elevation provided by the abduction tendency of these muscles. Part (c), though, shows a much reduced value of $\mathrm{GH}_{\mathrm{stab}}$, to less than 0.5 , indicating that the stability of the glenohumeral joint is maintained throughout the motion (with a factor of safety of 2).

Fig. 4 shows the effect of two different solvers on the stability of the model during the simulation. Part (a) shows that globally the two solvers result in the same motion given the same input activations. Part (b) shows a magnified $y$-axis to illustrate the more stable performance of the fourth-order Runge-Kutta solver as compared to the first-order Euler solver.

Table IV shows the performance of the model on the realtime system. The TET is the computation time required by the processor to complete one integration of the system. If the largest stable step size for the model is larger than this TET, then real-time operation is possible. The table shows that both solvers are capable of real-time operation with this model. (a)

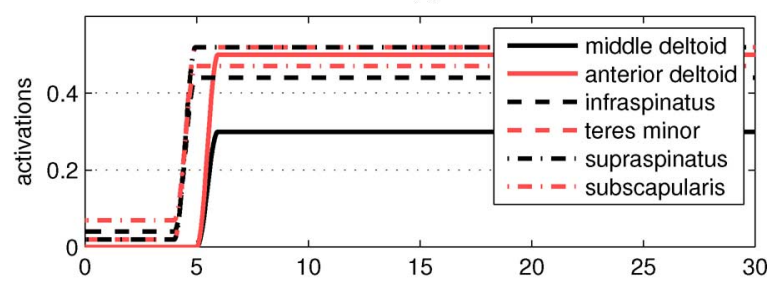

(b)

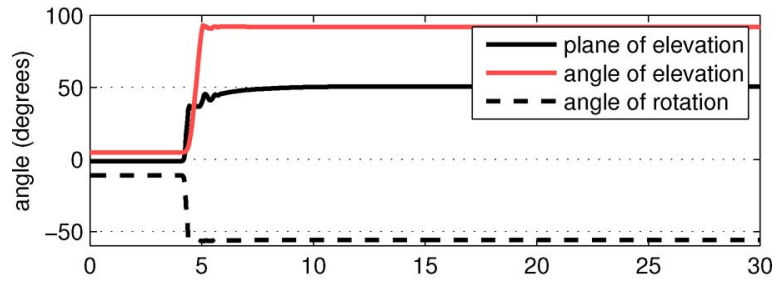

(c)

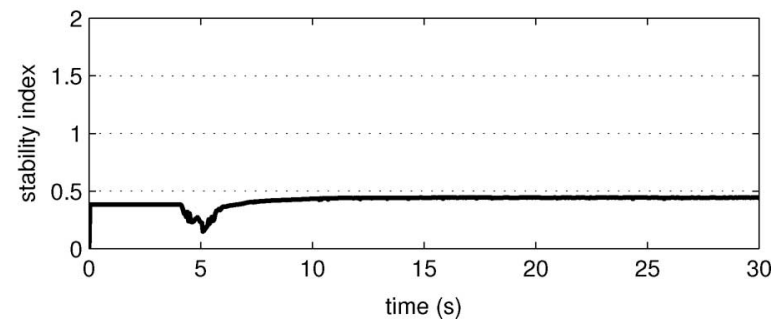

Fig. 3. Activation of the middle and anterior parts of the deltoid muscles, with increased stimulation of the rotator cuff muscles are shown in part (a). The resulting model motions are shown in part (b). Note the increased stability of the glenohumeral joint, shown by the stability value of $<0.5$ in part (c).

(a)

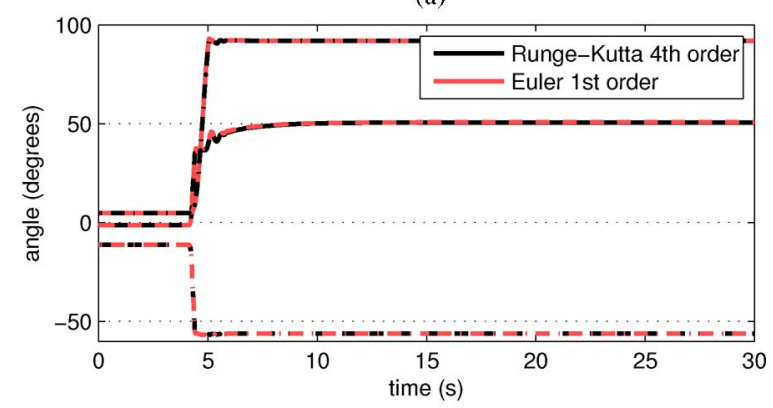

(b)

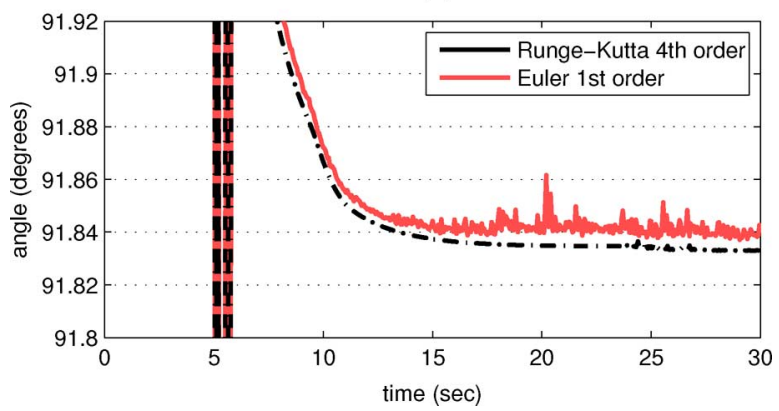

Fig. 4. Stability of the simulation with two different solvers at their minimum step sizes. Part (a) shows that the model response to the given input is extremely similar with the two solvers. Part (b) shows the increased stability of the RungeKutta fourth-order solver over the Euler first-order in a magnified new. 
TABLE IV

Performance of Model on ReAl-Time System (1.8-GHz PentiUm IV), AVERAGED ACross THREE TASKS

\begin{tabular}{|c||c||c|}
\hline Solver & Task Execution Time & Stable Step Size \\
\hline Runge-Kutta 4th order & $0.51 \mathrm{~ms}$ & $1.0 \mathrm{~ms}$ \\
\hline Euler 1st order & $0.13 \mathrm{~ms}$ & $0.5 \mathrm{~ms}$ \\
\hline
\end{tabular}

\section{DISCUSSION}

\section{A. Model Evaluation}

The goal of this study was to develop a real-time musculoskeletal model of the human upper limb for use in a dynamic arm simulator. The real-time model described in this study is based on anatomical parameters and structures described in [20] and previously implemented in a large-scale model using SIMM [22]. Verification of the model response to muscle activation indicates that the model still behaves as expected, even after conversion of the data to the format suitable for real-time execution. The primary outcome of this paper is thus the real-time performance allowing the model to be used in "patient-in-the-loop" testing of neural prosthesis systems.

The muscle stimulation experiments showed that the model responds in a predictable way to various levels of muscle stimulation. The model is stable in the resting position with only low-level baseline activation of the rotator cuff muscles to stabilize the glenohumeral joint, and is also stable in arbitrary intermediate positions of elbow flexion, forearm pronation, and humeral abduction (Figs. 1 and 2). Furthermore, glenohumeral stability is maintained during humeral abduction by activation of the rotator cuff muscles, and is seen to be compromised when these muscles are not concurrently activated (Figs. 2 and 3). The main outcome of these experiments, though, is that all these movements were successfully simulated in real time.

\section{B. Computational Performance}

Simulink offers a range of fixed-step solvers for use with xPC Target, from first to fifth order. The first-order Euler solver has the lowest computational cost, but also requires the smallest step size to maintain a given level of accuracy. The Runge-Kutta fourth-order solver, in contrast, has a higher computational cost, but produces smaller errors for a given step size. This means that the higher order solver is able to perform the simulation with a larger step size, permitting a larger TET while still running in real time. This is the tradeoff that must be assessed in the evaluation of the solvers. The solvers at intermediate orders to those described were not found to offer any performance benefit, and so were not used. The best solver choice is also influenced by the range of stiffnesses encountered in the system.

In our system, high stiffness is introduced by the combination of high-stiffness muscle elements (those with short series elastic components, i.e., short tendon slack lengths such as pronator quadratus) and low-inertia degrees of freedom, such as forearm pronation/supination. This stiffness requires the use of the small step sizes seen in Table IV to ensure a stable solution. The Runge-Kutta fourth-order solver was found to be the best compromise between speed and accuracy for the integration of this system. This solver allowed real-time operation of the model on a 1.8-GHz Pentium IV with a solver step size of $1 \mathrm{~ms}$. The Euler first-order solver was also able to run in real time, with a step size of $0.5 \mathrm{~ms}$, but the simulation was slightly less stable in that case, as seen in Fig. 4.

In our experience, calculation of the muscle elements is the most time-consuming part of the simulation, and additional degrees of freedom in the kinematics are less significant. We therefore expect some increase in simulation time with a more complex model comprising more muscle elements, but this tends to increase linearly with the number of polynomial terms used to model the muscles and the number of muscle elements. We are therefore confident that the whole arm model, which is the long-term goal of this work, will also be suitable for real-time simulation, since the increase in complexity should be a factor of around 2 or less.

\section{Model Limitations}

The model is a fully 3-D representation of the glenohumeral joint and elbow that includes all the relevant muscles and degrees of freedom. As far as can be seen from the literature, this is the only model of this scale whose ability to run in real time has been tested. However, our model does have the limitation of the fixed scapula. This simplification was used to allow us to develop the real-time framework without jumping straight into the complexity of the closed-chain shoulder girdle and focus on necessary developments such as monitoring and ensuring glenohumeral stability. This limits the range of motion of the arm somewhat, as the shoulder girdle would normally contribute to the range of motion of the arm. This also has the effect of increasing the length range over which some of the muscles have to operate, as the glenohumeral joint must provide all the movements that would otherwise be provided by the combination of scapular and humeral motion. The model still operates under these conditions, providing a real-time, 3-D simulation of arm movement, but the motions at individual joints may be slightly less realistic than they would be with a full model including a shoulder girdle.

The anatomical parameters in the model are taken from measurements based on a single cadaver, but our model can use any internally consistent parameter set describing the shoulder and elbow mechanism. Many other models described in the literature are based on the averaging of all available data (for example, [16]) or parameter optimization (for example, [26]), both of which have their merits. Using single cadaver measurements maintains relationships between structures, such as muscle attachment points, moment arms, optimum fiber lengths, etc., which may be lost using other methods. For real-time, "patientin-the-loop" experiments, matching the model to the patient through the use of actual patient data such as muscle points of origin and insertion would be very attractive. This is not really feasible at this time, but may become so in the future with improvements in imaging modalities. Despite differences between the actual subject and the model, the real-time simulator still provides an extremely useful tool that allows us to examine how well a user can control a complex neuroprosthesis and what the 
TABLE V

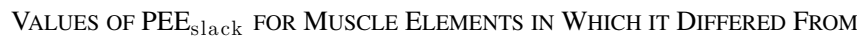
Default Value of 1.0 (NoRmalized to Optimum Fiber LenGth)

\begin{tabular}{|c|c|c|}
\hline Muscle name & Element no. & $P E E_{\text {slack }}$ \\
\hline Infraspinatus & {$\left[\begin{array}{lll}4 & 5 & 6\end{array}\right]$} & {$\left[\begin{array}{lll}1.1 & 1.2 & 1.1\end{array}\right]$} \\
\hline Teres minor & {$\left[\begin{array}{lll}1 & 2 & 3\end{array}\right]$} & {$\left[\begin{array}{lll}1.3 & 1.4 & 1.4\end{array}\right]$} \\
\hline Supraspinatus & [1] & [1.1] \\
\hline Biceps, long head & {$[1]$} & {$[1.3]$} \\
\hline Biceps, short head & {$\left[\begin{array}{ll}1 & 2\end{array}\right]$} & {$\left[\begin{array}{ll}1.5 & 1.6\end{array}\right]$} \\
\hline Triceps, long head & {$\left[\begin{array}{llll}1 & 2 & 3 & 4\end{array}\right]$} & {$\left[\begin{array}{llll}1.5 & 1.5 & 1.4 & 1.2\end{array}\right]$} \\
\hline Triceps, medial head & {$\left[\begin{array}{lllll}1 & 2 & 3 & 4 & 5\end{array}\right]$} & {$\left[\begin{array}{llllll}1.3 & 1.3 & 1.4 & 1.3 & 1.2\end{array}\right]$} \\
\hline Brachialis & {$\left[\begin{array}{lllllll}1 & 2 & 3 & 4 & 5 & 6 & 7\end{array}\right]$} & {$\left[\begin{array}{lllllll}1.2 & 1.2 & 1.1 & 1.4 & 1.3 & 1.2 & 1.3\end{array}\right]$} \\
\hline Brachioradialis & {$\left[\begin{array}{lll}1 & 2 & 3\end{array}\right]$} & {$\left[\begin{array}{lll}1.2 & 1.2 & 1.3\end{array}\right]$} \\
\hline Pronator teres & {$[1]$} & [1.3] \\
\hline Supinator & {$\left[\begin{array}{llll}2 & 3 & 4 & 5\end{array}\right]$} & {$\left[\begin{array}{llll}1.2 & 1.3 & 1.2 & 1.3\end{array}\right]$} \\
\hline Pronator quadratus & [1] & [1.1] \\
\hline Triceps, lateral head & {$\left[\begin{array}{llll}1 & 2 & 3 & 4\end{array}\right]$} & {$\left[\begin{array}{llll}1.2 & 1.2 & 1.2 & 1.2\end{array}\right]$} \\
\hline
\end{tabular}

effects of different command interfaces and controllers are on that control.

\section{Applications}

A realistic 3-D model of the human upper limb that provides real-time feedback on arm motion allows us to perform experiments with the patient in the loop in a way that would not otherwise be possible. For example, in the development of control algorithms for implanted FES systems, a variety of virtual systems can be investigated before any actual FES intervention is applied to the subject. Simulation of different muscle groups can be tried, stimulation levels can be tested, and controller designs can be developed. The level of control that a potential user would have with that system can be assessed with minimal risk and inconvenience to the subject. In the field of BMIs, decoding algorithms can be tested to assess the feasibility of multi-DOF control and fundamental questions about control mechanisms can be answered for conditions that would not otherwise be possible.

\section{CONCLUSION}

We have developed a real-time, 5-DOF, dynamic model of the human shoulder and elbow. This model differs from previous models of these joints in that it can compute the dynamics for an appropriate time duration faster than that time duration, i.e., in "real time." This was accomplished by replacing computationally demanding calculations (muscle moment arms and muscle lines of action) by efficient, precomputed polynomial fits. Our model achieved real-time performance using very modest computing hardware, demonstrating the utility of our approach and providing ample opportunities for increasing the complexity of the model in the future.

This study demonstrated the feasibility of producing a model of sufficient complexity that would run in real time, a prerequisite for the DAS, which will allow the execution of "patientin-the-loop" experiments for FES command and control development. Future work will focus on the integration of the shoulder girdle and hand to the model, i.e., additional degrees of freedom will be added to model the sterno-clavicular and acromio-clavicular joints, and a simple hand model will be added. This will form a complete upper arm simulator for the design and testing of neural prosthesis controllers and BMIs.

\section{APPENDIX}

\section{MUSCLE STIFFNESS PARAMETERS}

Table $\mathrm{V}$ shows the values of $\mathrm{PEE}_{\text {slack }}$ (parallel elastic element slack length) that were modified from the default value of 1.0 to give reasonable resting lengths for the muscles based on limb resting position.

\section{REFERENCES}

[1] M. W. Keith, P. H. Peckham, G. B. Thrope, K. C. Stroh, B. Smith J. R. Buckett, K. L. Kilgore, and J. W. Jatich, "Implantable functional neuromuscular stimulation in the tetraplegic hand," J. Hand Surg. [Amer.], vol. 14, pp. 524-530, May 1989.

[2] A. M. Bryden, W. D. Memberg, and P. E. Crago, "Electrically stimulated elbow extension in persons with $\mathrm{c5} / \mathrm{c} 6$ tetraplegia: A functional and physiological evaluation," Arch. Phys. Med. Rehabil., vol. 81, pp. 80-88, Jan. 2000.

[3] R. Thorsen, R. Spadone, and M. Ferrarin, "A pilot study of myoelectrically controlled FES of upper extremity," IEEE Trans. Neural Syst. Rehabil. Eng., vol. 9, no. 2, pp. 161-168, Jun. 2001.

[4] M. R. Popovic, D. B. Popovic, and T. Keller, "Neuroprostheses for grasping," Neurol. Res., vol. 24, pp. 443-452, Jul. 2002.

[5] K. T. Ragnarsson, "Functional electrical stimulation after spinal cord injury: Current use, therapeutic effects and future directions," Spinal Cord, vol. 46, no. 4, pp. 255-274, Sep. 2007.

[6] R. Rupp and H. J. Gerner, "Neuroprosthetics of the upper extremityClinical application in spinal cord injury and challenges for the future," Acta Neurochir. Suppl., vol. 97, pp. 419-426, 2007.

[7] R. Kirsch, A. Acosta, F. van der Helm, R. Rotteveel, and L. Cash, "Modelbased development of neuroprostheses for restoring proximal arm function," J. Rehabil. Res. Dev., vol. 38, pp. 619-626, Dec. 2001.

[8] M. Ferrarin, F. Palazzo, R. Riener, and J. Quintern, "Model-based control of FES-induced single joint movements," IEEE Trans. Neural Syst. Rehabil. Eng., vol. 9, no. 3, pp. 245-257, Sep. 2001.

[9] J. P. Giuffrida and P. E. Crago, "Functional restoration of elbow extension after spinal-cord injury using a neural network-based synergistic FES controller," IEEE Trans. Neural Syst. Rehabil. Eng., vol. 13, no. 2, pp. 147-152, Jun. 2005.

[10] B. P. Heilman and R. F. Kirsch, "Model-based development of a user control algorithm for postural control via a FES-based standing neuroprosthesis," in Proc. Conf. Proc. IEEE Eng. Med. Biol. Soc., 2004, vol. 6 , pp. 4614-4617.

[11] T. Pistohl, T. Ball, A. Schulze-Bonhage, A. Aertsen, and C. Mehring, "Prediction of arm movement trajectories from ecog-recordings in humans," J. Neurosci. Methods, vol. 167, pp. 105-114, Jan. 2008.

[12] D. M. Taylor, S. I. H. Tillery, and A. B. Schwartz, "Direct cortical control of 3-D neuroprosthetic devices," Science, vol. 296, pp. 1829-1832, Jun. 2002.

[13] J. Carmena, M. Lebedev, R. Crist, J. O’Doherty, D. Santucci, D. Dimitrov, P. Patil, C. Henriquez, and M. Nicolelis, "Learning to control a brainmachine interface for reaching and grasping by primates," PLoS Biol., vol. 1, no. 2, p. e42, Nov. 2003.

[14] F. C. van der Helm, "A finite element musculoskeletal model of the shoulder mechanism," J. Biomech., vol. 27, pp. 551-569, May 1994.

[15] B. A. Garner and M. G. Pandy, "Musculoskeletal model of the upper limb based on the visible human male dataset," Comput. Methods Biomech. Biomed. Eng., vol. 4, pp. 93-126, Feb. 2001.

[16] K. R. S. Holzbaur, W. M. Murray, and S. L. Delp, "A model of the upper extremity for simulating musculoskeletal surgery and analyzing neuromuscular control," Ann. Biomed. Eng., vol. 33, pp. 829-840, Jun. 2005.

[17] I. W. Charlton and G. R. Johnson, "A model for the prediction of the forces at the glenohumeral joint," Proc. Inst. Mech. Eng. [H], vol. 220, pp. 801-812, Nov. 2006.

[18] M. Hauschild, R. Davoodi, and G. E. Loeb, "A virtual reality environment for designing and fitting neural prosthetic limbs," IEEE Trans. Neural Syst. Rehabil. Eng., vol. 15, no. 1, pp. 9-15, Mar. 2007. 
[19] R. Davoodi, C. Urata, M. Hauschild, M. Khachani, and G. E. Loeb, "Model-based development of neural prostheses for movement," IEEE Trans. Biomed. Eng., vol. 54, no. 11, pp. 1909-1918, Nov. 2007.

[20] M. D. Klein Breteler, C. W. Spoor, and F. C. Van der Helm, "Measuring muscle and joint geometry parameters of a shoulder for modeling purposes," J. Biomech., vol. 32, pp. 1191-1197, Nov. 1999.

[21] I. Gûnal, N. Köse, O. Erdogan, E. Göktürk, and S. Seber, "Normal range of motion of the joints of the upper extremity in male subjects, with special reference to side," J. Bone Joint Surg. Amer., vol. 78, pp. 1401-1404, Sep. 1996.

[22] D. Blana, J. G. Hincapie, E. K. Chadwick, and R. F. Kirsch, "A musculoskeletal model of the upper extremity for use in the development of neuroprosthetic systems," J. Biomech., vol. 41, pp. 1714-1721, 2008.

[23] J. M. Winters and L. Stark, "Analysis of fundamental human movement patterns through the use of in-depth antagonistic muscle models," IEEE Trans. Biomed. Eng., vol. BME-32, no. 10, pp. 826-839, Oct. 1985.

[24] S. G. McLean, A. Su, and A. J. van den Bogert, "Development and validation of a 3-D model to predict knee joint loading during dynamic movement," J. Biomech. Eng., vol. 125, pp. 864-874, Dec. 2003.

[25] R. F. Kirsch, D. Boskov, and W. Z. Rymer, "Muscle stiffness during transient and continuous movements of cat muscle: Perturbation characteristics and physiological relevance," IEEE Trans. Biomed. Eng., vol. 41, no. 8, pp. 758-770, Aug. 1994.

[26] B. A. Garner and M. G. Pandy, "Estimation of musculotendon properties in the human upper limb," Ann. Biomed. Eng., vol. 31, pp. 207-220, Feb. 2003. 